\title{
SECONDARY USE OF CLINICAL DATA FOR SCIENTIFIC RESEARCH PURPOSES IN THE DITAS PROJECT
}

\author{
Paola Aurucci, Ilio Catallo, Mariet NouriJanian, Andrea Micheletti and Alberto Sanna \\ Center for Advanced Technology in Health and Wellbeing, IRCCS San Raffaele Hospital, Milan, Italy
}

\begin{abstract}
Thanks to the advent of the digital revolution, coupled with advances in computing power, medical research is becoming increasingly data intensive. Extracting meaningful information from data sets and continuous flood of data is a challenge but holds unparalleled potential for observational studies on, e.g., the use and safety of drugs and rare diseases, epidemiological studies and digital disease detection. At the same time Cloud computing is used for two main reasons in secondary health research: first, to allow large-scale digital health data processing using readily scalable, external infrastructure; and second, to allow the sharing of digital health data with collaborators via a jointly usable IT environment. In this context of the DITAS project (https://www.ditas-project.eu/), to reconcile the often-competing values of data protection and innovation, aims at providing developers with processes and tools for the creation of GDPR compliant data-intensive applications in cloud computing. The aim of this paper is to show how in DITAS context researchers can lawfully further process for research purposes sensitive data originally collected for clinical activity when anonymization (Section 2) and pseudonymization (Section 3) techniques are applied.
\end{abstract}

\section{KEYWORDS}

Data Protection, Cloud, DITAS, Medical Research, Anonymization, Pseudonymization

\section{INTRODUCTION}

Over the past twenty-five years, the digital revolution has made it possible for healthcare facilities and health agencies to use massive digital databases for the storage of administrative and health service data gathered from routine clinical practice [Stenardo et al. 2013].

These databases, despite being designed and alimented for service-related organizational purposes, have proved to be useful in observational biomedical research [Romio et al. 2013].

Indeed, the number of studies that are based on these "secondary" data sources increased [Thiese 2014] [Sorense 1996]. In the last ten years, the availability of such a large group of heterogeneous data-sets, coupled with advances in computing power, is driving researchers to create sophisticated algorithms for the analysis of pre-existing and real-time data valuable to medical research (not previously combinable through matching techniques), to look for patterns, correlations, and links of potential significance [Mostert et al. 2016] and even to predict the likelihood of events based on continuous data stream through the application of machine learning techniques (Big Data Analytics) [Mantelero 2017]. Extracting meaningful information from data sets and continuous flood of data is a challenge but holds unparalleled potential for observational studies on, e.g., the use and safety of drugs and rare diseases, epidemiological studies and digital disease detection. Cloud computing is used for two main reasons in secondary health research: first, to allow large-scale digital health data processing using readily scalable, external infrastructure; and second, to allow the sharing of digital health data with collaborators via a jointly usable IT environment [Molnár-Gábor et al. 2017]. Yet, whilst the possibilities in terms of innovative research springing from "general analysis" continue to expand, developments in IT and the utilization and reutilization of sensitive data have led to increasing concern about the effectiveness of data protection regulations still based on "notice and consent or anonymization paradigm", which is challenged in the context of data intensive medical research [Mostert et al. 2016]. On the other hand, concerns regard also the utilization of clouds, since their limitations in data security and in

\footnotetext{
${ }^{1}$ Recital 29, GDPR, available at: 〈http://data.europa.eu/eli/reg/2016/679/oj>.
} 
respecting the rights of patient donors and research participants [Molnár-Gábor et al. 2017]. In the wake of a growing need for consistent protection of personal data that should not hinder responsible technological research of Big Data through manifold techniques, such as machine learning [Pagallo 2017], the European Parliament and the Council in 2016 adopted the much-awaited "Regulation on the protection of natural persons with regard to the processing of personal Data and on the free Movement of such data" (from now on "GDPR"). ${ }^{2}$ This act repealed Directive 95/46/EC and entered in force on May 2018. This two-year grace period was supposed to allow Member States to revise or adapt their legislation in order to come into compliance with the GDPR [Piciocchi et al. 2017]. The GDPR carves out numerous derogations for "historical or scientific purposes" [Maldoff 2016], also called "research exemption", to avoid restrictions on secondary uses of sensitive data (Article 5(1)(b), Recital 50). However, these derogations depend on the appropriate safeguards set up by the data controller in keeping with recognized ethical standards [Maldoff 2016], which lower the risks of discrimination or stigmatisation. Article 89(1) specifies that one way for the controller to comply with the duty to apply appropriate safeguards - where anonymization contrasts with the purposes of the processing [Bolognini and Bistolfi 2017] - concerns the use of "pseudonymization". The use of this strong de identification technique however is not enough and must be combined with additional technical and organizational measures such as security measures for the conservation of the secret key used for the encryption or hash function, the principle of privacy-by-design, privacy-by-default, transparency and the presence of a data protection officer [Bolognini and Bistolfi 2017]. For the sake of illustration, in the following sections we consider how such data protection aspects have been addressed in the context of the DITAS project (https://www.ditas-project.eu/), a H2020 European project that aims at providing developers with processes and tools for the development of GDPR compliant data-intensive applications in cloud computing. In particular, we examine the case of a medical research institute that would like to provide its researchers with a digitalized data sharing platform for the access to clinical data. Ideally, the platform should assist researchers in expressing their need for research data and provide an immediate way of downloading data of interest. At the same time, researchers should be prevented from accessing patients' personal data while carrying out their research activities. DITAS attempts to reconcile these two contrasting objectives. It does so by mediating the access to the clinical data, so as to put in place pseudonymization (or - when necessary, anonymization) techniques. Hence, researchers never get direct access to clinical data. Instead, DITAS provides a uniform entry-point to the data, and decides, depending on the person requesting the access, what kind of transformation should be applied to the data prior to their delivery. The aim of this paper is to show how in DITAS context researchers can lawfully further process for research purposes sensitive data originally collected for clinical activity when anonymization (Section 2) and pseudonymization (Section 3) techniques are applied. Since GDPR crows back the power to EU Member States, allowing them to introduce further safeguards with regard to the processing of genetic data, biometric data or data concerning health, in the Conclusion the paper aims to restrict the focus of analysis to the Italian legal framework that on this specific subject is a real "Dedalus"3 of different inputs and rules [Piciocchi et al. 2017]. The Italian scenario shows that delegation of powers back to the national legal systems of the Member States entails a number of critical drawbacks, like hampering the progress of cross-border medical research, and preventing the achievement of the second - but of equal importance - goal of the GDPR: to guarantee the "free movement of personal data".

\section{ANONYMIZATION CHOICE: USE CASE 1}

GDPR applies to "personal data," which is defined, in part, as "any information relating to an identified or identifiable natural person". ${ }^{4}$

\footnotetext{
${ }^{2}$ Regulation (EU) 2016/679 of the European Parliament and of the Coucil of 27 April 2016 on the protection of natural persons with regard to the processing of personal data and on the free movement of such data, and repealing Directive 95/46/EC (General Data Protection Regulation), L 119/1, available at: <http://data.europa.eu/eli/reg/2016/679/oj>.

${ }^{3}$ In Greek Mythology Deadalus was a skillful craftsman which was imprisoned within Daedalus the labyrinth he created.

${ }^{4}$ Article 4(1), GDPR, available at: <http://data.europa.eu/eli/reg/2016/679/oj>.
} 
The final text of the GDPR should be a cause for celebration by the medical research community for two reasons: first, scientific research is defined broadly in the final text, including fundamental research, applied research and privately funded research and - second, it grants some exemptions from its requirements when personal data are processed for scientific research [Dove 2018].

However, GDPR continues to clearly favor scientific research that uses anonymized data ${ }^{5}$ [Penasa 2018]. Indeed, Article 89(1) states that, if the purposes of the scientific research can be achieved using these data, then it can be fulfilled by further processing which does not permit or no longer permits the identification of data subjects". "Datum which have been anonymized is no longer "personal data" and is therefore not subject to the requirements of data protection law. In the context of the use case n. 1 of the DITAS project, researchers can access anonymized nutritional data, which have been collected as part of the day-to-day clinical activity, and that can also serve as a valuable source of information for conducting, e.g., statistical studies on the population. Researchers can then use this data collected during the clinical practice for new research purposes, unknown at the time of the collection, without the need to respect GDPR provisions, usually trigger by the use of personal data or personal identifiable information [Vayena and Gasser, 2016]. Furthermore, in the scientific research context the use of anonymization technique is then functional not only for the storage of data, but also for enabling the use of historically collected data for secondary research, their eventual sharing with third parties, and potential linkage to other datasets. For this reason, use case n. 3 of DITAS project provides that researchers external to the research institute can only access anonymized data, dispensing them to respect GDPR requirements governing data sharing [Vayena and Gasser, 2016]. The Article 29 Working Party (WP29) 2014 in his Opinion on Anonymization Techniques ${ }^{7}$ set a high bar for what qualifies as fully anonymized data. WP29 states that, taking into account all means "likely reasonably" to be used to re-identify the data, anonymization must be "irreversible" and "as permanent as erasure." 8 At the same time, according to Article 29 WP29 there are many anonymization techniques ${ }^{9}$ but it also recognized that the risk of re-identification of each of these technique is never zero, since they present risk in terms of inference and linkability. ${ }^{10}$ It is fundamental then to establish what constitutes "an acceptable low level of risk". Recital 26 GDPR states that "account should be taken of all means reasonably likely to be used (...) to identify the natural person either directly or indirectly". This language suggests a "reasonable acceptable standard of anonymization" should be preferred to the "impossible standard". In any case, to anonymize data, it is necessary to proceed with an ex ante risk of identification assessmen [Bolognini and Bistolfi 2017] that must be context and technology dependent. According to Recital 26 the risk re-identification should be analyzed considering different factors such as time, effort or researches needed to re-identify data, the purpose of the processing, the risk of external attack and the available technology at the time of the processing. Furthermore, even if the risk of re-identification of an anonymized dataset is calculated to be acceptably small at the time of data disclosure, potential linkage of that dataset to others at an unknown future point in time may increase the possibility of identifying the person or of inferring information about him/her connecting different data and trace them back to a single subject. This is why, as required in the resents Guidelines on the protection of individuals with regard to the processing of personal data in a world of Big Data ${ }^{11}$, data controllers "shall regularly review the assessment of the risk of re-identification". Much of the literature have, in fact, shows that in the era of big data with potentially multiple data linkage opportunities and new analytical methods, e.g. data mining, increase the risk of re-identification [de Montjoye et al. 2015].

\footnotetext{
${ }^{5}$ Recital 26, GDPR, available at: 〈http://data.europa.eu/eli/reg/2016/679/oj>.

${ }^{6}$ Article 89(1), GDPR, available at: 〈http://data.europa.eu/eli/reg/2016/679/oj>.

Article 29 Working Party, Opinion 05/2014 on anonymization techniques, 10 April 2014, available at: https://ec.europa.eu/justice/article-29/documentation/opinion-recommendation/index_en.htm.

${ }^{8}$ Id., at 6.

${ }^{9}$ Id., at 12 .

${ }^{10}$ Id., at $12-17$

${ }^{11}$ Council of Europe, Guidelines on the protection of individuals with regard to the processing of personal data in a world of Big Data, available at: $\langle$ https://rm.coe.int/16806ebe7a $>$.
} 


\section{THE PSEUDONIMIZIATION CHOICE: USE CASE 3}

As seen in the previous paragraph GDPR continues to clearly favor scientific research that uses anonymized data.

However, when this could also not be possible, since currently popular method of redaction of any information which may increase the risk of re-identification of the individual can greatly compromise the scientific utility of the data.

Furthermore, potential linkage of that dataset to others and the use of analytics [Mantalero 2017] will increase the number of attributes will need to be considered in the assessment of risk, rendering any calculation of this risk of reidentification difficult or potentially unknowable. In these cases scientific research may be carried out using personal data. To lawfully process personal data for scientific research purposes at least the following legal elements must be considered in advance: a) the respect of principles set by Articles 5 and b) The presence of legal grounds for data processing c) the obligation to inform data subjects according to Articles 13-14 GDPR. In order to reconcile the often-competing values of data protection and innovation, in all this three set of rules, the GDPR carves out numerous derogations (also called "research exemptions") for "historical or scientific purposes" [Chassang 2017], allowing researchers to avoid restrictions on secondary uses of health data. These "research exemptions" will be analyzed in the following paragraphs. In the particular case of the DITAS project, researchers internal to the research institute are allowed to access pseudonymized data, whereas external researchers can only access anonymized data. DITAS transparently applies the proper transformation depending on the kind of person that initiates the data request, thus greatly simplifying the provisioning of a data sharing platform for a research institute

\subsection{GDPR Research Exemptions to the General Principle}

Starting from the general principles, the "purpose limitation" principle of Article 5 (1)(b) - which is of fundamental importance in the general application of data protection rules and compliance with these rules - requires that specified, explicit and legitimate purposes should be known and defined at the moment of data collection and that data must not be further processed in a way that is incompatible with those purposes. In the contest of modern observational medical research - where sensitive data are constantly shared, aggregated and reused - it is for the controller increasingly hard to predict at the time of data collection all the specific future purposes of investigations [Mittelstadt et al. 2016]. To re-contact each individual data subject to obtain explicit consent or to inform her/him on the new research purpose is often impractical, prohibitively expensive and could undermine the validity of outcomes [Vayena and Gasser, 2016]. The possibility to predict at the time of the collection future possible purposes of research it becomes impossible when researches imply the use of AI techniques - like machine learning - that are hypothesis-generating rather than hypothesis-testing research. This is because the aim of these techniques is to discover unforeseen connections between data points, that cannot be accurately predicted prior to using the data. In order to facilitate of secondary observational research - where health data are further processed for purposes that are different from those for which they were originally collected - Article 5(1)(b) also provides that further processing for archiving purposes in the public interest, scientific or historical research purposes shall not be considered incompatible with the initial purposes. This language is reflected in Recital (50) that goes even further, stating that further processing for scientific research should in any case be considered "compatible lawful processing". This recital also provides the possibility of using the original data subject's consent, or another legal basis that has allowed the original collection when the purpose of the new processing (further processing or secondary use) is "compatible" with the purposes initially declared. This presumption of compatibility with the purposes announced at the time of the collection is related to the exemption to the principle of "storage limitation" which allows personal data to be stored for longer periods insofar as the personal data will be processed solely for archiving purposes in the public interest, scientific or historical research purposes or statistical purposes. Both articles, however, specify that this presumption of compatibility with the initial purposes is not fully automatic, but requires a data controller and a data processor to implement appropriate safeguards designed to protect the data subject and to reduce risks in accordance with Article 89(1). Article 89(1) specifies that the purpose of this safeguards is to ensure that specific technical and organizational measures are in place in particular in order to ensure respect for the 
principle of data minimization. The use of "pseudonymization" techniques allows the controller to ensure respect for the principle of data minimization (where anonymization contrasts with the purposes of the processing as seen at the beginning of the paragraph). Recital 29 specify that when pseudonymization is used to make "general analysis" legitimate it must be combined with additional security measures to reduce the risk of reidentification [Bolognini and Bistolfi, 2017]. Just to name a few: technical measures to store the cryptographic system or hash function separately from the data in order to prevent the pseudonym from being attributed to the original data, measure to protect the places where they are stored from undue access, authentication mechanisms (ID and password biometrics or both) to to allow (researchers) or prevent (unauthorized persons) access to the keys or to correlation tables. This presumption of compatibility has been subject to restrictive interpretation the European Commission which in the draft Q\&A analyzing the interplay between the EU Clinical Trials Regulation ("CTR") and the EU General Data Protection Regulation ("GDPR") excluded the presumption of compatibility provided under Article 5(1)( b) in all circumstances. However, following the interpretation of the European Data Protection Board (from now on EDPB) on this draft, the EC has highlighted in the new Q\&A that secondary use of clinical trial data for scientific research purposes is by default compatible with its original use, in accordance with Article 5(1)(b) of the GDPR. As a result, it should not be necessary to obtain a new consent in order to engage in additional secondary research.

\subsection{GDPR Research Exemptions to the Legal Basis for Processing}

Respecting fundamental principles of data protection listed under Article 5 is not enough for lawfully processing personal data. The controller - before starting the processing - must find one or more legal grounds (legitimate basis) for data processing, listed under Article 6. However, primary and secondary medical research implies the use of genetic data, biometric data, data concerning health, which are traditionally held to be particularly sensitive type of personal data, necessitating stricter condition for its processing. For this reason, GDPR requires that for the processing of these special categories of data, the legal basis identified under Article 6 shall be applied only if Article 9 provides for a specific derogation from the general prohibition to process this data (i.e. exceptions to the general prohibition). While patient consent provides both a lawful basis and a permitted condition for processing personal data collected in the context of a health, it is one only of the available options, and others may be more appropriate. The EU's data protection advisory body, the Article 29 Working Party (WP29, now the European Data Protection Board), issued its finalised Guideline on the concept of consent under the GDPR in mid-April 2018 (the EU Guidelines) ${ }^{12}$. The EU Guidelines explain that under the GDPR, consent is not always required to process data for the purpose of scientific research-for example, processing may be lawful on the basis that it is necessary for the performance of a task carried out in the public interest, for compliance with a legal obligation, or for the legitimate interests of the data controller or a third party, as long as appropriate safeguards are in place and the processing is fair, lawful, transparent and accords with data minimisation standards and individual rights, and that similar considerations also apply to the processing of special category data under the scientific research condition (Article 9(1)(j)). However, s seen in the previous paragraph further processing of clinical data for scientific research purposes is by default compatible with its original use, in accordance with Article 5(1)(b) of the GDPR. As a result, it should not be necessary to obtain a new consent in order to engage in additional secondary research

\subsection{GDPR Research Exemptions to the Obligation to Inform Data Subjects}

To respect general fundamental principles and to ensure a valid legal grounds/lawfulness conditions of data processing could not be enough. A general rule, coming from Articles 13-14 of the GDPR, imposes to adequately inform data subjects about their personal data processing, even in case of further processing, no matter whether a new legal basis is required or not. This is the "privacy notice" or "privacy policy" that we are used to receive as data subjects and users, almost in all cases personal data are going to be collected and processed (some exceptions occur, for instance, just in justice/law enforcement scenarios). Collecting data from the (dark, deep, surface) web, such as collecting data from publicly available records and documents, or

\footnotetext{
12 Article 29 Working Pary, Guidelines on consent under Regulation 2016/679, available at: https://ec.europa.eu/newsroom/article29/item-detail.cfm?item_id=623051
} 
from another hospital, implies that data are not obtained directly from the data subject. In this case (collection not from the data subject, but from third parties), Article 14.5.b) of the GDPR applies and justify at certain conditions even the exclusion of the obligation for the data controller to inform data subjects, where and insofar as "the provision of such information proves impossible or would involve a disproportionate effort, in particular for processing for archiving purposes in the public interest, scientific or historical research purposes or statistical purposes, subject to the conditions and safeguards referred to in Article 89(1) or in so far as the obligation referred to in paragraph 1 of this Article is likely to render impossible or seriously impair the achievement of the objectives of that processing. In such cases the controller shall take appropriate measures to protect the data subject's rights and freedoms and legitimate interests, including making the information publicly available. However, the only exception to a controller's Article 13 information obligations, where it has collected personal data directly from a data subject, occurs "where and insofar as, the data subject already has the information " (Article 13(4)). The WP29 in its Guidelines on transparency under Regulation $2016 / 679^{13}$ notes that this exception should be construed narrowly, and the principle of accountability requires controllers to demonstrate and document precisely what information the data subject has, how and when they received it and that no changes have occurred to that information that would render it out of date. This is a problem Scientific Institute for Research, Hospitalization and Health Care, like those involved in DITAS use case 3. In this case due to the instrumental nature of the activity of health care provided by the aforementioned institutions with respect to research, the further processing for research purposes is carried out not by a third party, but by the same controller that collected the data in the first place for providing medical assistance. This imply that controller should recontact and inform the patient each time he/her datum are going to be used for a scientific research purpose that was not specified at the moment of the collection. It seems here to be room for the use Recital 33, which recognize the difficulty to predict all possible specific research purposes at the time of data collection and seems to allow for a broader consent to certain areas of scientific research and for participants to select those areas. However, the Article 29 Working Party in its Guideline on consent down play Recital 33 to the point of becoming nearly non-existent. More clarity must be provided at European level on this topic.

\section{CONCLUSION}

The previous Sections focus on which GDPR requirements should be respected in order to lawfully further processing sensitive data in medical observational studies.

This is fundamental in order to reach the aim of the DITAS project: providing developers with processes and tools for the development of GDPR compliant data-intensive applications in cloud computing.

However, although the GDPR is intended favor scientific research and to reduce inconsistencies in the implementation of data protection across the EU, it crows back powers to EU Member States. In line with Article 9(4) of the GDPR - that allows Member States to introduce further safeguards with regard to the processing of genetic data, biometric data or data concerning health [Pagallo 2017] - the recently reformed Italian Data Protection Code ("IDPC"), as amended by Legislative Decree no. 101/2018, adds and requires further conditions in order to lawfully process secondary health related data for scientific purposes without consent or information to data subjects. At present terms, the relevant legal framework applying to processing data for medical research in Italy is a complex combination of IDPC's provisions, old and new deontological rules, and old and "updated" general Authorizations of the Italian Data Protection Authority (from now on "IDPA" or "Garante"). Furthermore, Article 22(11) of the Legislative Decree no. 101/2018 says that rules concerning the processing of genetic data, biometric data and data concerning health, of the previous Code of Privacy (Legislative Decree no. 196/2003) continue to apply, if compatible with the GDPR, until the biannual guidance on the processing of these data, foreseen in in Article 2 septies of the IDPC, will be provided by the Garante. This document (biannual guidance) will identify the safeguard measures (including security measures, such as encryption and pseudonymization) to apply for the processing of biometric, genetic and health-related data, having regard to the specific purposes of processing. This provision of the IDPC appears in contradiction with the principle of self-accountability of data controllers

\footnotetext{
${ }^{13}$ Article 29 Working Pary, Guidelines on transparency under Regulation 2016/679, available at:

https://ec.europa.eu/newsroom/article29/item-detail.cfm\%3Fitem_id\%3D622227+\&cd=4\&hl=it\&ct=clnk\&gl=it
} 
(i.e., no ex ante identification of the proper safeguards to apply for a specific research purposes but ex post onus on data controllers to demonstrate compliance with the GDPR in case of breach). However, until this document won't be issued by the Garante, it is not clear which rules actually applies to the secondary processing of sensitive data for scientific research purposes. The only thing that is sure is that fragmentation of data protection legislation inside EU, and particularly strict requirements for lawfully processing of data for health research (which creates further burdens for data controllers and processors) may hamper the responsible data sharing that DITAS project aims to provide, and consequently, the free movement of personal data within the internal market. ${ }^{14}$ The hope is that emerging code of conducts at European level, encouraged by the GDPR in Articles 40 and 41, may enable harmonization in the application of data protection law in this specific field [Marelli and Testa, 2018]. Initiatives already exist to promote code of conducts on the large-scale re-use and linkage of health-related and genomic data. ${ }^{15}$ On the other hand, code of conducts at National level may also constitute a valid point of reference for the scientific community to understand how to be compliant and a possibility for research organizations, patient advocacy groups, and industrial representative [Marelli and Testa, 2018] to agree on practical data protection rules that will meet the needs of the sector as well as the requirements of the GDPR.

\section{ACKNOWLEDGEMENT}

This work is supported by European Commission H2020 Programme through the DITAS Project no. 731945.

\section{REFERENCES}

Barfield et. al., 2018. Research handbook on the law of artificial intelligence. Title (in italics). Edward Elgar Publishing, Cheltenham, UK. Northampton, MA, USA.

Bodorik P. et al, 1991. Deciding to Correct Distributed Query Processing. In IEEE Transactions on Data and Knowledge Engineering, Vol. 4, No. 3, pp. 253-265.

Bolognini L. et al., 2017. Pseudonymization and impacts of Big (personal / anonymous) Data processing in the transition from the Directive 95 / 46 / EC to the new EU General Data Protection Regulation. Comput Law Secur Rev Int J Technol Law Pract, Vol. 33, No. 2, pp. 171-81.

Chassang G., 2017, The impact of the EU general data protection regulation on scientific research. Ecancermedicalscienc, Vol. 11, No. 709, pp. 1-12.

De Montjoye Y. A. et al., 2015. Unique in the shopping mall: on the reidentificability of credt card data. Science, Vol. 347, No. 6221, pp. 536-539.

Dove E. S., 2019, The EU General Data Protecion Regulation: Implication for International Scientific Research in the Digital Era. Journal of Law, Medicin \& Ethics, Vol. 46, No. 4, pp. 1-32.

Mantelero A., 2017. Regulating Big Data. The guidelines of the Council of Europe in the Context of the European Data Protection Framework. Computer Law and Security Review, Vol. 33, No 5, pp. 584-602.

Marelli L. and Testa G., 2018, Scrutinizing the EU General Data Protection Regulation, Science Vol. 360, No. 6388, pp. 496-498.

Molnár-Gábor F. et al., 2017. Computing patient data in the cloud: practical and legal considerations for genetics and genomics research in Europe and internationally. Genome Medicine, Vol. 9, No. 58, pp. 1-12.

Mondschein C.F. et al. 2019, The EU's General Data Protection Regulation (GDPR) in a Research Context. In: Kubben P. et al. (eds) Fundamentals of Clinical Data Science. Springer, Cham, pp. 55-71.

Mostert M. et al., 2016. Big Data in medical research and EU data protection law: challenges to the consent or anonymise approach". Eur J Hum Genet, Vol. 24, No. 7, pp. 956-960.

Pagallo U., 2017. The Legal Challenges of Big Data: Putting Secondary Rules First in the Field of EU Data Protection. European Data Protection Law Review, Vol 3, No. 1, pp 36-46.

Penasa S. et al., 2018. The EU General Data Protection Regulation: How will it impact the regulation of research biobanks? Medical Law International, Vol. 18, No. 4, pp. 1-15.

\footnotetext{
${ }^{14}$ Recital 13 of the GDPR.

${ }^{15}$ BBMRI-ERIC, Code of conduct for health research, available at: http://code-of-conduct-for-health-research.eu/
} 
Piciocchi C. et al., 2018. Legal issues in governing genetic biobanks: The Italian framework as a case study for the implications for citizen's health through public-private initiatives. J Community Genet, Vol. 9, pp. 177-90.

Romio S. et al., 2013. Real-World Data From the Health Decision Maker Perspective. What Are We Talking About?, Epidemiology, Biostatistics and Public Health, Vol 10, No. 3, e8979.

Stendardo A. et al., 2013. Legal aspects regarding the use and integration of electronic medical records for epidemiological purposes with focus on the Italian situation. In Epidemiology Biostatistics and Public Health, Vol. 10, No. 3, e8971.

Thiese M. S., 2014. Observational and interventional study design types; an overview. Biochem Med (Zagreb), Vol. 24, No. 2, pp. 199-210. 